\title{
DVC analysis of a polymer material subjected to tensile loading with synchrotron radiation tomography
}

\author{
Sooyoung Lee, Wooseok $\mathrm{Ji}^{*}$ \\ Department of Mechanical Engineering, Ulsan National Institute of Science and Technology (UNIST), Ulsan, South Korea
}

\section{A R T I C L E I N F O}

\section{Keywords:}

Digital volume correlation

Synchrotron radiation

In situ testing

Computed tomography

Subsurface deformation

\begin{abstract}
A B S T R A C T
Subsurface deformation behavior of a polymeric material is studied through the digital volume correlation (DVC) technique. Fundamental principles of the DVC technique are presented and the supplemental state-of-the-art algorithmic schemes to improve the efficiency and accuracy of the DVC analysis are also introduced. Tensile tests on an epoxy material are performed in conjunction with synchrotron radiation tomography. In order to create randomly distributed grayscale values in the tomograms for the following image analysis, microscale highdensity particles are embedded when the epoxy specimens are fabricated. 3D tomographic images taken at multiple loading steps are utilized for the DVC analysis. The performance of the present DVC analysis is evaluated with the experimental data.
\end{abstract}

\section{Introduction}

Since the 1980s, the digital image correlation (DIC) technique has been widely used to measure real-time full-field data pertaining to displacement and strains [1-5]. This non-destructive testing method is capable of measuring deformation behavior of a material over a wide area in exceptional detail. The capability of measuring out-of-plane displacement is also incorporated [6-10] into the method, which conventional measuring tools such as a strain gage and an extensometer fail to offer. As a result, nowadays, the imaging technique is routinely used for the quantitative investigation of material behavior. However, the DIC technique can measure the displacement and strain data only on the specimen surface where a speckle pattern is applied. The surface information is often insufficient to completely represent complicated mechanical response of an anisotropic heterogeneous material like composites or a material with a complex microstructure such as bones and foams. In order to have a better understanding of these types of materials, it is necessary to observe subsurface deformation behavior, which the current DIC approach cannot detect [11].

Digital volume correlation (DVC) technique has been effectively used to determine the internal volumetric deformation behavior of solid materials since it was proposed by Bay and Smith [12,13]. The DVC technique can be considered as the extended version of the DIC approach to three-dimensional (3D) domain in conjunction with micro-computed tomography $(\mu \mathrm{CT})$. Therefore, the $3 \mathrm{D}$ imaging technique shares its simplicity in terms of principles and effectiveness when it comes to applications with the well-established DIC method [14,15]. Since the DVC technique was first applied in the field of medical biology, various materials including bones [12,16], soft materials [17,18], wood [19], sand [20], and ceramic [21] are characterized by the 3D image analysis. As the $\mathrm{X}$-ray $\mu \mathrm{CT}$ technique evolves, the resolution of tomographic images has been refined as low as one micron with better image quality, lower noise, and fewer artefacts. The subsurface deformation behavior of a material can now be quantified at a micro-length scale through the DVC approach. However, the computational burden of the DVC analysis is considerably heavier than the DIC approach because of the additional degree of freedom (DOF) in space. Thus, the improvement of the computational speed has been one of the key issues in the 3D DVC analysis, posing a challenge to researchers from the past decade [15].

In the present study, deformation behavior inside a polymeric material is investigated using the DVC technique. 3D CT images for the DVC analysis are taken during in situ tensile tests performed at a synchrotron facility. The in situ mechanical testing protocol using the synchrotron light source is described in great detail. Polymeric materials are generally considered as an isotropic homogeneous material. Tomograms of such a material may not have a wide variation of grayscale values that is crucial for image correlation techniques. Note that the DIC analysis cannot be performed without a speckle pattern. The DVC analysis typically utilizes image contrast mainly resulted from the natural pattern of a complex material microstructure. Since the polymer

\footnotetext{
* Corresponding author.

E-mail address: wsji@unist.ac.kr (W. Ji).
} 


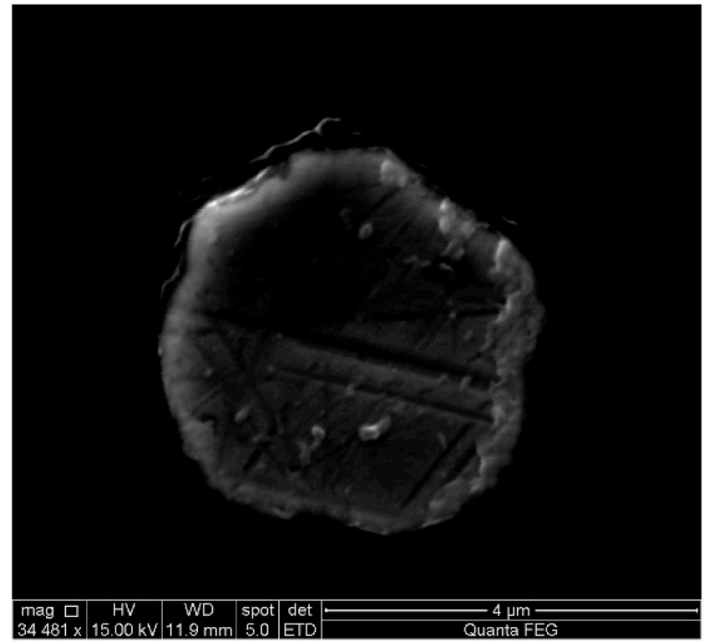

Fig. 1. Magnified view of a $\mathrm{Cu}$ particle embedded in the epoxy specimen.

specimen considered in the present study does not have such a distinct natural pattern, high-density microscale particles are inserted when the specimen is fabricated to create a "3D volumetric speckle pattern." The present DVC analysis is performed using in-house DVC software optimized for massive 3D volume data with micro-voxel resolution. The matching process of the software is based on the zero-normalized cross correlation (ZNCC) method. The DVC program employs fast Fourier transform (FFT) and precomputation strategies, which are classical remedies to improve the computational efficiency for recursive template matching processes. Parallelization scheme is also implemented for the present DVC analysis.

The rest of this paper is organized as follows. Section 2 describes the specimen preparation process and in situ mechanical testing protocol. Section 3 first presents the fundamental principle of the correlation process in the view of the DIC technique and then describes the details of the present DVC approach including the resolution refinement technique. The present DVC analysis is evaluated in Section 4 by comparing the measurement results against experimental data. The conclusions of the present study are summarized in Section 5.

\section{Experimental procedure}

\subsection{Preparation of specimens}

For the demonstration of the present DVC analysis, the INF-114 epoxy resin with the INF-212 hardener from Pro-Set Inc. is utilized to fabricate test specimens. INF-114/INF-212 is an ordinary resin system for a typical infusion molding process. In the present work, the resin is mixed with the hardener by hand and the mixture is dripped into an acrylic mold with a rectangular shape using a conventional liquid dropper. The resin is then gelated at room temperature and subsequently post-cured for $8 \mathrm{~h}$ at $80 \mathrm{C}$ in an oven to improve the mechanical performance as instructed by the technical data sheet [22]. The specimen dimension is $25 \mathrm{~mm} \quad 3 \mathrm{~mm} \quad 2 \mathrm{~mm}$ with the gage length of $11 \mathrm{~mm}$. In order to detect the subsurface deformation behavior of the epoxy using the image correlation approach, micro-scale copper particles are embedded into the specimens to introduce heterogeneity to the homogeneous material and thus to create the gray-level distribution in X-ray images [23]. Embedding the copper particle in the material is analogous to applying a speckle pattern for the DIC technique. Since the density of copper is much higher than that of the epoxy resin, the particles will absorb more X-rays and thus will be imaged in bright color on radiographs. Fig. 1 shows a copper particle embedded in the specimen. Typical diameter of the sphere-shaped particles is about $4 \mu \mathrm{m}$. The copper particles and epoxy resin are mixed using a centrifugal paste mixer, and a three roll mill is used for dispersing the particles in the viscous resin as evenly as possible. The three roll mill is a machine whereby a shear force is applied via three horizontally positioned rolls rotating in opposite directions at different speeds relative to each other, in order to mix, refine, disperse, or homogenize viscous materials fed into it [24].

The amount of the copper particles inserted into the resin is set to $20 \mathrm{wt} \%$, which is equivalent to $5.88 \%$ by the measure of a volume fraction. The particular weight percentage is decided by observing the section images of the specimens containing different percentages of the particles. Fig. 2 (a), (b) and (c) show the SEM images of the specimens with the particle mass fractions of 10,15 , and $20 \mathrm{wt} \%$, respectively. The black region corresponds to the epoxy resin and the red dots represent the copper particles, which are originally white but later colored red for clear visibility. As can be seen in Fig. 2 (a), (b) and (c), the particles are well dispersed in the epoxy resin for all the cases. However, the distributions with the mass fractions of 10 and $15 \mathrm{wt} \%$ seem quite sparse for the image correlation process and, thus, the mass fraction is set to $20 \mathrm{wt}$ $\%$ in the present experimentation.

\subsection{In situ tension test setup}

Fig. 3(a) shows the typical scheme of synchrotron radiography. As the X-ray beam penetrates the sample, X-ray photons are absorbed by the material. The absorption rate of the $\mathrm{X}$-rays varies, mainly depending on material density. The differences collected by the detector is utilized to create a radiograph to reveal the internal structure of the sample. In the synchrotron radiography, a number of projection images are taken at multiple angles by rotating the sample from 0 to 180 as illustrated in Fig. 3(a). The series of radiographs are then further processed to reconstruct tomograms as shown in Fig. 3(b) and the sliced images are stacked up along the rotation axis of the sample to render the 3D volume data of the sample.

In the present study, X-ray scanning is performed while the epoxy polymer sample is loaded in order to examine internal response of the

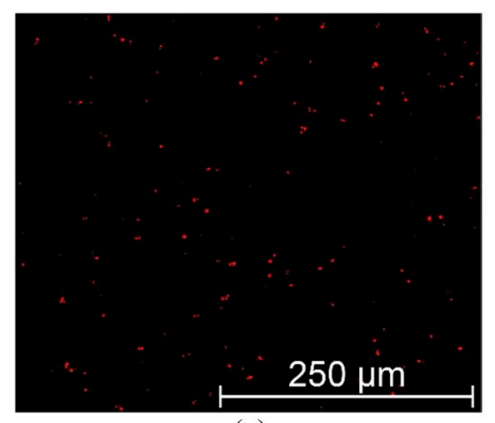

(a)

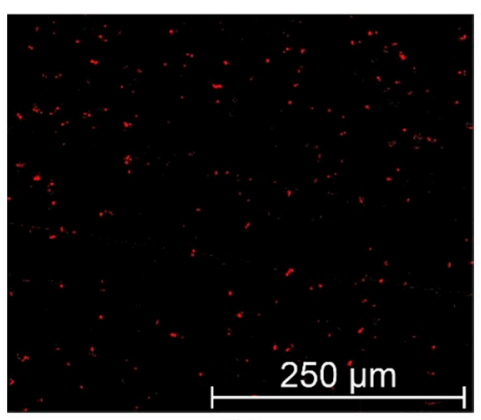

(b)

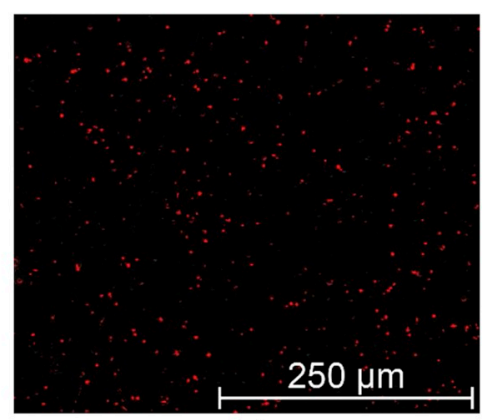

(c)

Fig. 2. Section images of the specimens with various particle mass fractions (a) $10 \mathrm{wt} \%$ mass fraction (b) $15 \mathrm{wt} \% \mathrm{mass}$ fraction (c) $20 \mathrm{wt} \% \mathrm{mass}$ fraction. 


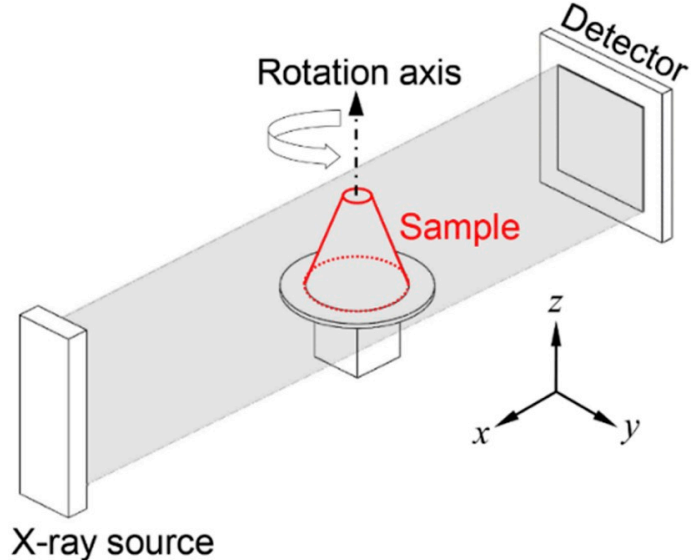

(a)

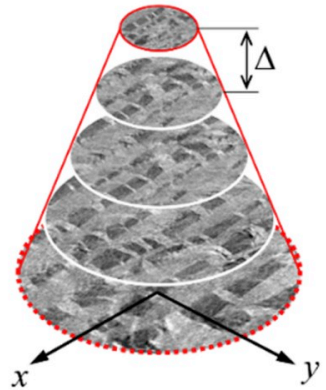

(b)

Fig. 3. Schematic illustration of the X-ray scanning process and the reconstructed tomograms.

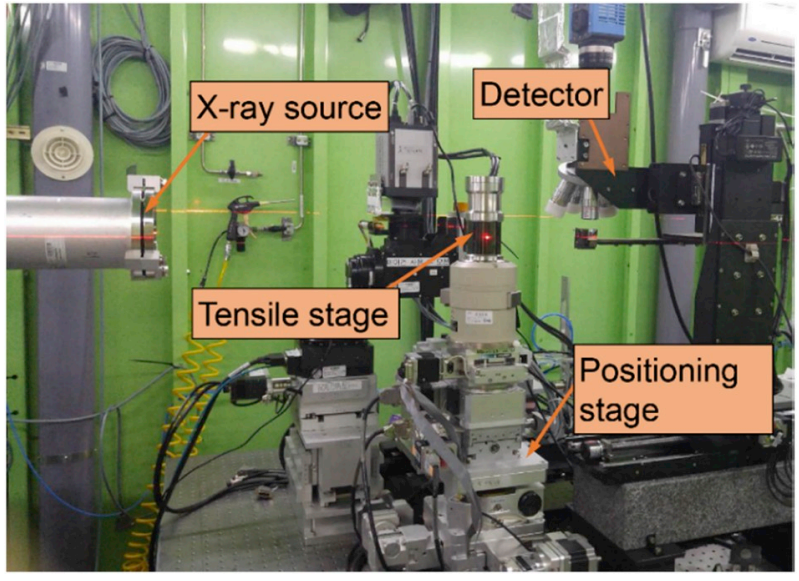

Fig. 4. Experimental setup at $6 \mathrm{C}$ Beamline of PAL.

material in situ. The subsurface deformation behavior of the epoxy material is imaged using a synchrotron light source at Beamline 6C in Pohang Accelerator Laboratory (PAL). Fig. 4 shows the general experimental setup at the beamline. The loading device is mounted on a motorized positioning stage to precisely locate the sample with respect to the beam path. The specimen-to-detector distance (SDD) is set to

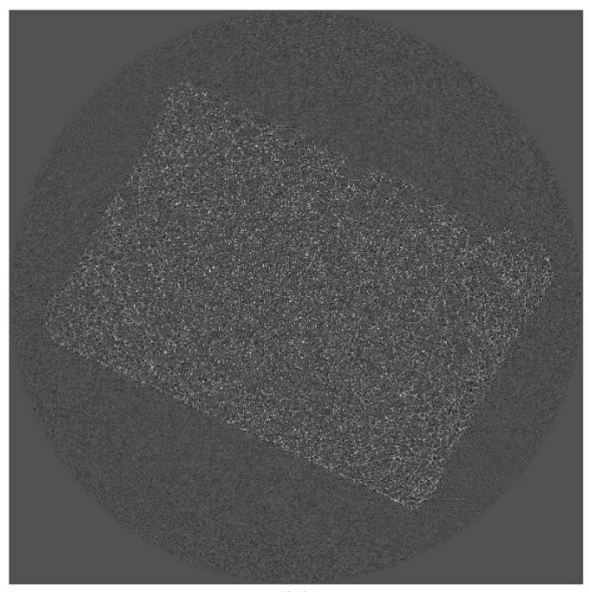

(a)
$35 \mathrm{~mm}$, which is the shortest available distance at the beamline. SDD should be short to enhance the spatial resolution of X-ray images while long SDD improves image contrast [25]. The detector system consists of a scintillator and a camera with a microscope lens. In the present experiment, we use a $6-\mu \mathrm{m}$ thick LSO:Tb scintillator layer, an sCMOS camera with a 25602160 pixels readout and a 4 microscope lens. The detector setup results in the field of view (FOV) having the size of $4.163 .51 \mathrm{~mm}^{2}$ for the projectional radiography with the effective resolution of $1.625 \mu \mathrm{m} /$ pixel. The monochromatic parallel X-ray beam from the synchrotron light source is modulated at a photon energy of $25 \mathrm{keV}$. The energy level is high enough to penetrate the sample at least $10 \%$ in transmission, but low enough to result in strong signal-to-noise ratio for good image quality [25].

In situ tension tests are performed using the microtest tensile stage (Deben Ltd., UK) as shown in Fig. 4. Tensile loading is applied to the specimen by a stepper motor, while the top and bottom of the specimen is fastened with serrated grips in the tensile stage. Force data is measured by a load cell with a capacity of $5 \mathrm{kN}$ and a linear extensometer is utilized to measure the crosshead displacement. The loading device is equipped with a tubular glassy-carbon window with the diameter of $59 \mathrm{~mm}$ and the thickness of $3 \mathrm{~mm}$. The window is strong enough to support the structure of the tensile stage during the tension test and thin enough to transmit a sufficiently large number of X-ray photons with the transmission rate of $78 \%$ at the photon energy level of $25 \mathrm{KeV}$. In the present study, X-ray scanning is carried out at 20 loading steps with the interval of $10 \mathrm{~N}$ from 0 to $190 \mathrm{~N}$. When the force value

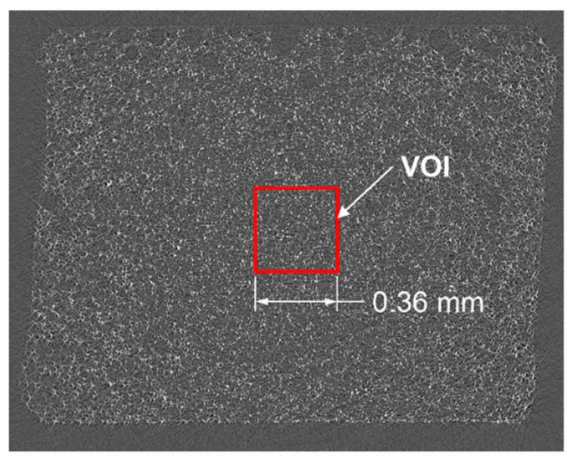

(b)

Fig. 5. Tomograms of the epoxy specimen (a) Raw image (b) Cropped and rotated image for DVC analysis with the definition of a VOI region. 


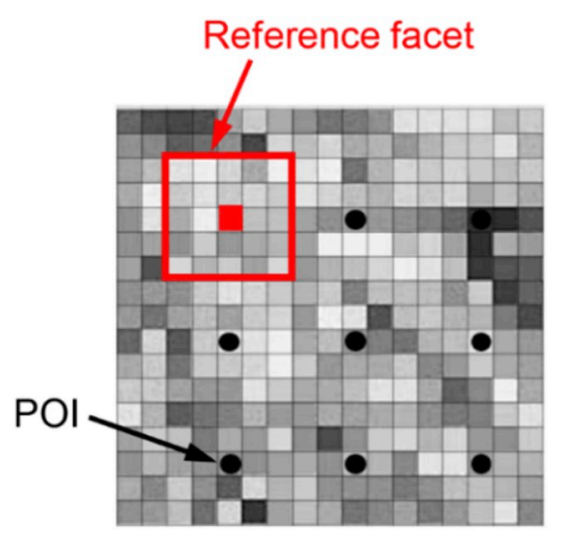

(a) Reference image

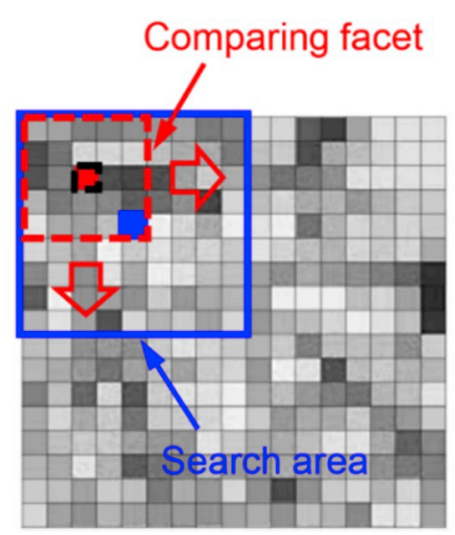

(b) Target (deformed) image

Fig. 6. Schematic illustration of the correlation process between the two images.

measured by the load cell reaches the target load, the motor is paused to start X-ray imaging. At each loading step, 1200 projection images are acquired while the sample is rotating from 0 to 180 with the exposure time of $0.5 \mathrm{~s}$ per projection. Total measurement time taken at each loading step is about $18 \mathrm{~min}$.

A stack of tomograms is constructed from the radiographs using a commercial reconstruction software, Octopus (XRE, Gent, Belgium) with a filtered back-projection (FBP) reconstruction algorithm. 2160 tomograms are obtained at each loading step with a pixel resolution of 25602560 per image, in which the physical length of one pixel is $1.625 \mu \mathrm{m}$. Fig. 5(a) is an example of the tomograms that shows the crosssection of the epoxy polymer specimen. All the tomograms are cropped and rotated first accordingly as shown in Fig. 5(b) to reduce the image size for efficient DVC analysis.

\section{Digital volume correlation technique}

\subsection{Correlation principle between images}

As mentioned in the prior section, the DVC technique can be considered as a straightforward extension of the well-established DIC technique [14,15]. Therefore, for the efficient explanation of the present DVC analysis, the core principle of the general DIC approach is briefly described here. In principle, the DIC approach tracks small motions of randomly distributed points (speckle pattern) on the surface of a specimen from a series of images taken while the specimen is being deformed. The measured movements of the points are then utilized to compute the deformation behavior of the specimen. The motion tracking is essentially a correlation process between the reference and target images. Prior to the correlation, the points of interest (POIs) in the region of interest (ROI) are defined first. For example, the circular black dots, shown in Fig. 6, represent the POIs. The POIs in the reference (undeformed) image and the target (deformed) image are located on the same global coordinates. A "facet," which is a group of several pixels, with its center conforming to a POI is then constructed in the reference image. In the deformed state image, a searching area larger than the reference facet is assigned at the corresponding POI. The comparing facet is moving inside the searching window typically by one pixel at a time and the similarities between the reference facet and the trial facets are quantified using various template matching criteria.

The DIC method originally owes its name to the use of the crosscorrelation method for the matching criteria. In addition to the crosscorrelation (CC) criterion, various matching criteria such as the sum of absolute difference (SAC) and sum of squared difference (SSD) methods have been used for DIC analysis [1,26-28]. Some studies [29,30] have compared displacement and strain fields obtained from different matching criteria. The results show that the matching criteria principally yield the same results under the condition that the intensity values of each pixel remains unchanged in the deformed image. However, the intensity of the deformed image is susceptible to undesired changes in practical experiments. For example, the lighting may fluctuate with time, and the reflectivity of a specimen surface may change because of the increase in the surface roughness when the specimen is being deformed [30]. Under such a condition, the performance of correlating subvolumes becomes poor and the error in the displacement measurement can be significant. Therefore, a robust correlation criterion must be used to compensate for the possible intensity discrepancy [29,31].

The zero-mean normalized cross correlation (ZNCC) method is the most commonly used matching criterion owing to its insensitivity to the potential scale and offset changes in the intensity of the deformed image $[30,32,33]$. In the ZNCC method, for each searching area, the quantified similarities between the reference facet and the trial facets are assembled into a matrix. The components of the matrix are correlation coefficients expressed as

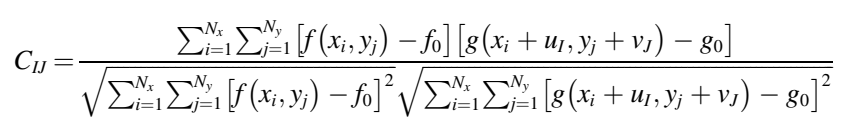

where $I$ and $J$ denote the current location of the moving facet within the search area in terms of pixel numbers in the horizontal and vertical directions, respectively. For example, in Fig. 6, the comparing facet can move by up to five pixels both in the horizontal and vertical directions, and thus, the integers $I$ and $J$ range from 1 to 5 . The correlation coefficient of the comparing facet illustrated using a red dashed line in Fig. 6 corresponds to $C_{11} . N_{x}$ and $N_{y}$ are the pixel numbers of the facet in the horizontal and vertical directions, respectively. $x_{i}, y_{j}$ are the center point coordinates of a single pixel in the reference facet, and $f x_{i}, y_{j}$ is the grayscale value of the single pixel. $g x_{i} \quad u_{I}, y_{j} \quad v_{J}$ is the intensity value of a single pixel centered at the coordinates $x_{i} \quad u_{I}, y_{j} \quad v_{J}$ in the comparing facet, where $u_{I}$ and $v_{J}$ are the distances of the current position of the comparing facet from the center of the search area in the horizontal and vertical directions, respectively. $f_{0}$ and $g_{0}$ are the mean intensity values of all the pixels inside the facets in the reference and deformed states, respectively.

As the comparing facet moves in the search area with an increment of one pixel, the $I \quad J$ matrix $\mathbf{C}$ is filled with the corresponding correlation coefficients. The location corresponding to the maximum $C_{I J}$ in the matrix is considered to be the new position of the reference facet in the image of the deformed state. Consequently, the displacement vector $\boldsymbol{p}$ $\{u, v\}^{\mathrm{T}}$ of the reference facet is determined. The full-field displacement data in the ROI can be obtained after the same procedure is applied to all 


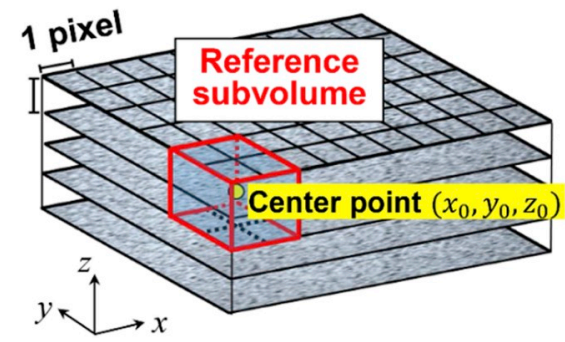

(a) $3 \mathrm{D}$ volume data in the reference state

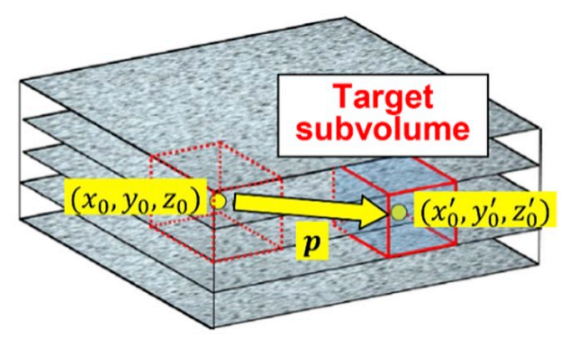

(b) Stack of tomograms in the deformed state

Fig. 7. Correlation process of the DVC approach.

the other POIs shown in Fig. 6. Note that the resolution of the computed displacement field depends on the number of POIs in the ROI.

The correlation coefficients $C_{I J}$ have a practical range of $0-1$, wherein 1 implies that two facets in the reference and deformed states are perfectly correlated. As mentioned previously, the ZNCC method is advantageous for image-processing applications wherein the brightness of the image or facet varies with the lighting or other experimental conditions $[30,32,33]$. The ZNCC method compensates for the discrepancy in the brightness between two comparing images through the normalization process as described in Eq. (1). In the normalization process, the mean intensity and the standard deviation of all the pixels inside the images (facets) are calculated. Subsequently, all the pixels inside the two images (facets) are subtracted by each mean value and finally divided by each standard deviation value.

The physical meaning of Eq. (1) can be explained by considering two comparing images (facets), which are subtracted by each mean intensity, as two vectors. The ZNCC equation is then considered as the inner product of the two vectors divided by the norms of each vector, which is equivalent to the cosine of the angle between the two vectors. Therefore, two vectors representing the reference and comparing facets are the same when the angle is zero, resulting in a correlation coefficient value of one.

\subsection{Correlation between $3 D$ CT images}

The correlation process in the DVC technique is based on the same principle of the DIC approach except that the DVC method deals with 3D volume data that are typically obtained through X-ray computed tomography (CT) as illustrated in Figs. 6 and 7. The DVC technique starts with defining cubical subvolumes, analogous to the facets in the DIC approach, as shown in Fig. 7(a). The similarity between the reference and target subvolumes are evaluated with a fully 3D-based correlation method. Eq. (1), defined in 2D space, is extended to 3D space for the DVC approach by including all the voxels inside the two subvolumes. Eq. (1) is then revised as artificial speckle pattern on the surface of a specimen. The right-hand side of Eq. (2) is based on the same principle as that of Eq. (1), except that the computation range is extended to the $3 \mathrm{D}$ space. As can be seen in Fig. 7(b), the DVC algorithm searches for the target subvolume in the deformed state using the greyscale value averaged over the subvolume. The increase in the computational workload during the correlation process is inevitable compared to the DIC technique, which leads to an increase in the total computation time. In the following section, the computation efficiency of the DVC approach is discussed.

\subsection{Enhancement of computational efficiency in DVC approach}

While the resolution of the displacement field computed from the DVC approach is directly affected by the discretization size of 3D volume data, a finer discretization leads to dramatically increased computational cost as a result of an increased number of correlation operations. Therefore, implementing an efficient correlation algorithm to achieve fast computational speed is one of the key issues in the DVC method. Simplifying the correlation process is one of the strategies of improving the computation efficiencies for both the DIC and DVC techniques. The standard cross-correlation (CC) method in conjunction with the fast Fourier transform (FFT) is now commonly used, taking advantage of the fact that the cross-correlation operation in the space domain is equivalent to the point-wise multiplication in the frequency domain $[15,30]$. This significant computation advantage is made possible by sacrificing the image matching performance. The CC-based image matching technique is incompetent in dealing with image noise and brightness discrepancies existing between two compared images $[15,27,29]$. 3D volume data obtained from $\mu \mathrm{CT}$ are usually much noisier than $2 \mathrm{D}$ images. Furthermore, the intensity levels of each voxel in the 3D image may change due to the deformation of a material especially when the images are taken during an in situ test. For these reasons, the ZNCC-based correlation approach is more appropriate than the CC method for the DVC analysis. However, the computational burden of using the ZNCC matching algorithm is more significant due to the

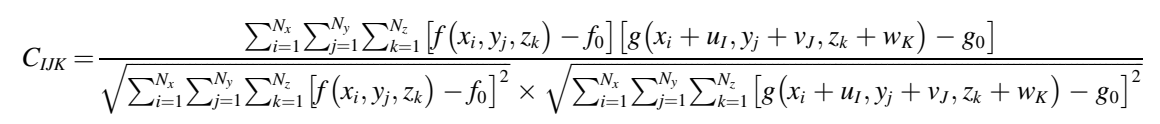

where $C_{I J K}$ is a correlation coefficient, $I, J$, and $K$ are integers indicating the current location of the compared subvolume in the search area along $x, y$, and $z$ directions, respectively. $f_{0}$ and $g_{0}$ are the mean intensity values of all the voxels inside the subvolumes corresponding to the reference and deformed states, respectively. The DVC method typically utilizes a natural microstructural pattern inside a sample to compute the intensity value of a subvolume while the DIC technique requires an additional computations of means and standard deviations of the grayscale images. The additional burden is traditionally relieved by utilizing a precomputed table data in conducting summations in Eq. (2) [18,34, 35]. Details about the FFT and sum-table approaches are described in Appendices A and B, respectively. In the present work, in addition to the two conventional remedies, simplified matching process and parallel computing are implemented to further improve the computational efficiency. 


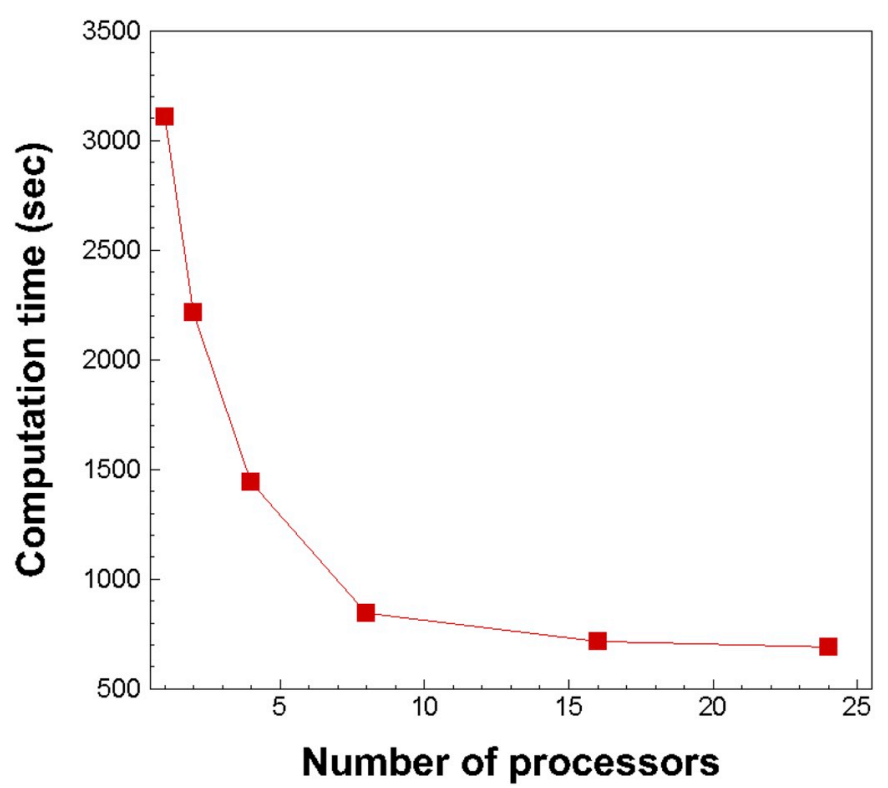

Fig. 8. Computation time for the correlation process of 12,348 subvolumes with different numbers of CPU cores.

The matching process between the reference and target subvolumes is simplified through the translation-only correlation approach. Since the specimen considered in the present study is a homogenous isotropic material and subjected to tensile loading only, any rigid body rotation of the subvolumes is negligible. Linearized small deformation theory is sufficient for the epoxy polymer within the strain range considered here. Several studies $[36,37]$ have considered adding a rotational DOF for each subvolume, especially for biomedical applications wherein a sample would undergo a large deformation. However, such an additional DOF can cause an increase in computational time during the correlation process, particularly with the increased number of subvolumes. Neglecting the rotational DOF may not be critical when tensile elongation behavior is dominant and a deformation increment between two comparing images remains small. Furthermore, the resolution of images obtained using the synchrotron light source is high enough for finer subvolume discretization. Since synchrotron radiography offers a superior image resolution with a finer pixel size $(1.625 \mu \mathrm{m}$ in the present work) than conventional medical CT scanners (15-82 $\mu \mathrm{m}$ [38]), the simplified matching process can be justified for the inorganic polymer specimen with finely discretized subvolumes.

Parallel computing is also implemented into the present DVC analysis. Since subvolume matching is a completely individual process, significant saving in computational cost can be achieved when multiple core processors simultaneously execute the correlation operations. In the present analysis, the iterative process to determine the entire correlation coefficients in VOI is parallelized through the built-in function, parfor, available in the Matlab toolbox. The runtime parallelization is evaluated with 12,348 cubical subvolumes when a single subvolume has a size of $3131 \quad 31$ pixels. Fig. 8 shows the performance evaluation results with a variance of processor numbers. As demonstrated in Fig. 8, the computational time is greatly reduced with an increased number of processors.

\subsection{Sub-voxel registration}

The smallest logical unit of a digital image is a picture element or pixel. The size of a pixel consequently determines the accuracy of displacement computed from DVC analysis since correlation resolution is confined in a discrete sense within a grid of pixels. However, a location change of a point in a deforming material is occurring in a continuous sense. It would be ideal to use images with higher resolution for the DVC analysis, but image resolution is often limited by an optical instrument and a data storage device. The limitation is typically overcome by implementing a sub-pixel registration algorithm [1,2,18,33,40,41]. This is a supplementary matching process to refine the correlation precision at the resolution finer than one-pixel size.

There are several sub-voxel registration approaches mostly used for the DVC technique. Peak-finding (PF) method [12,17,27] seeks a new peak position from a surface interpolating discrete correlation coefficient data. The surface typically interpolates the coefficient data of 333 or 555 voxels surrounding an initial peak position determined from a traditional DVC process. While the principle and implementation of the peak-finding scheme is simple, the resulting peak point may vary depending on interpolation models $[27,40]$. Coarse-fine searching (CFS) method interpolates discretely sampled intensity values and resamples the grayscale pattern with higher resolution for the following correlation process [1]. CFS is also based on the interpolation scheme and thus the calculation accuracy and convergence character are sensitive to the selection of interpolation function [40]. Newton-Raphson iterative (NRI) method models subvolume deformation using shape functions defined with displacement and its derivatives $[2,3,40]$. The unknown variables in the shape functions are determined when a similarity error between two comparing subvolumes is minimized. The minimization process results in a system of nonlinear equations, which can be solved by the Newton-Raphson method. CFS and NRI methods consider a shape change of a subvolume and apparently are not affected by large strains and rotations of deformed subvolumes [27]. Gradient-based (GB) method [40,41] is founded on the optical flow theory $[42,43]$ that calculates the velocity vectors of rigid objects from time-series images. The intensity function $g$ is linearized using a Taylor series with respect to the sub-voxel size displacement field $\Delta x, \Delta y$ and $\Delta z$. In doing so, the derivatives of the grayscale function, $g_{x}, g_{y}$ and $g_{z}$ are approximated following the similar approach in the optical flow theory. The only unknown variables $\Delta x, \Delta y$ and $\Delta z$ are determined through the minimization of the similarity error, resulting in a closed-form solution [40].

In the present study, the PF method is utilized to refine the correlation precision at the sub-voxel level due to its simplicity in implementation and effectiveness in calculation for the simple loading test considered here. Following the approaches of Bay et al. [12]. and Franck et al. [17], a quadratic polynomial is used to create a fitting contour of the discrete $C_{I J K}$ data. The correlation coefficients of $\begin{array}{lll}3 & 3 \text { voxels }\end{array}$ with the center corresponding to the initial maximum $C_{I J K}$ determined by the ZNCC-based analysis are interpolated by the quadratic polynomial function, $C x, y, z$, such that

$$
\begin{array}{cccccccc}
C x_{i}, y_{i}, z_{i} & a_{1} & a_{2} x_{i} & a_{3} y_{i} & a_{4} z_{i} & a_{5} x_{i} y_{i} & a_{6} y_{i} z_{i} & a_{7} z_{i} x_{i} \\
a_{8} x_{i}^{2} & a_{9} y_{i}^{2} & a_{10} z_{i}^{2} & i & 1,2, \ldots 27
\end{array}
$$

where $x_{i}, y_{i}$ and $z_{i}$ are the central coordinates of the 27 voxels and $a_{1}$ thru $a_{10}$ are the unknown polynomial coefficients to be determined. The coefficient can be found from the system of linear equations related to the 27 data points. In matrix form, $C_{I J K}$ at the 27 center points can be expressed as

$\mathbf{u} \mathbf{X A}$

where

u $\quad\left\{C x_{1}, y_{1}, z_{1}, C x_{2}, y_{2}, z_{2}, \ldots, C x_{27}, y_{27}, z_{27}\right\}^{T}$

$\mathbf{X}\left[\begin{array}{cccccccccc}1 & x_{1} & y_{1} & z_{1} & x_{1} y_{1} & y_{1} z_{1} & z_{1} x_{1} & x_{1}^{2} & y_{1}^{2} & z_{1}^{2} \\ 1 & x_{2} & y_{2} & z_{2} & x_{2} y_{2} & y_{2} z_{2} & z_{2} x_{2} & x_{2}^{2} & y_{2}^{2} & z_{2}^{2} \\ \vdots & \vdots & \vdots & \vdots & \vdots & \vdots & \vdots & \vdots & \vdots & \vdots \\ 1 & x_{27} & y_{27} & z_{27} & x_{27} y_{27} & y_{27} z_{27} & z_{27} x_{27} & x_{27}^{2} & y_{27}^{2} & z_{27}^{2}\end{array}\right]$ 


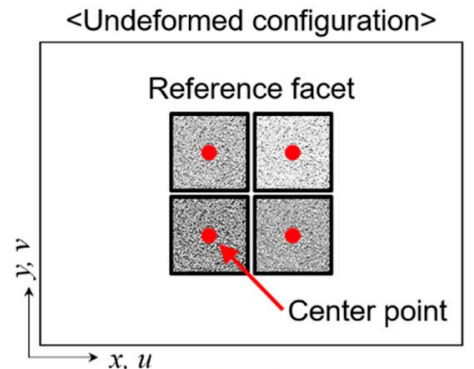

(a)

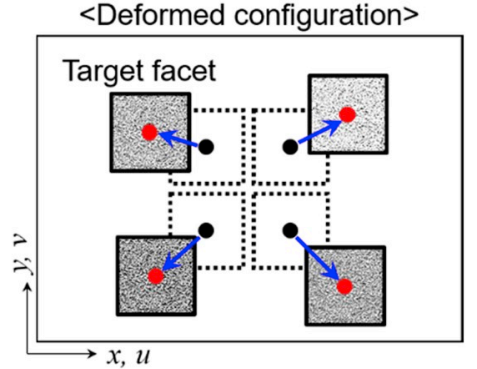

(b)

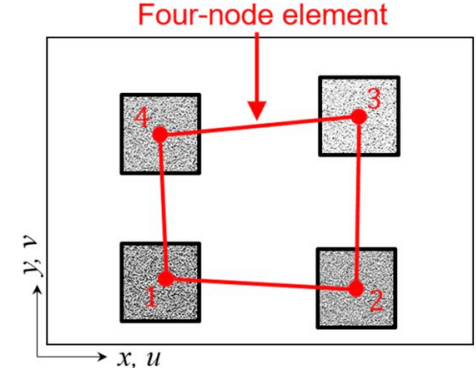

(c)

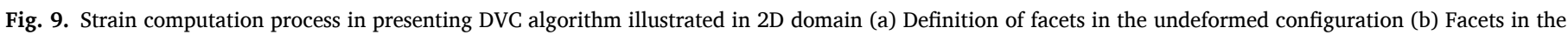

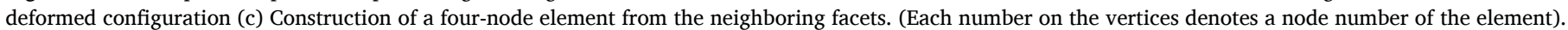

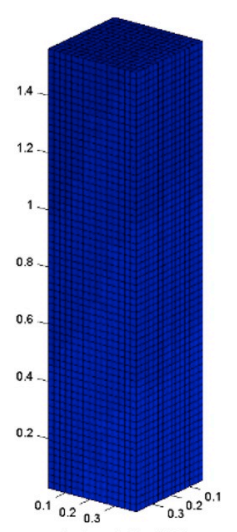

(a) $10 \mathrm{~N}$

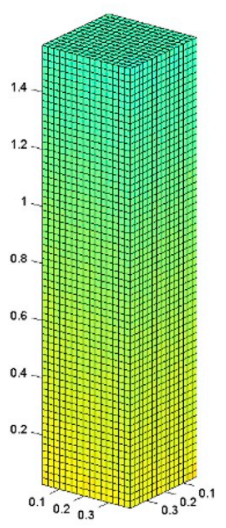

(f) $160 \mathrm{~N}$

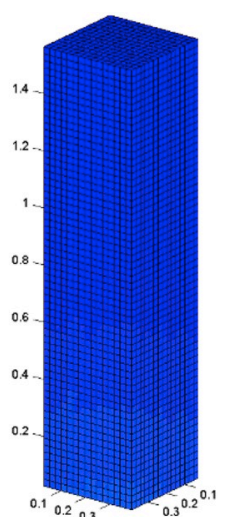

(b) $90 \mathrm{~N}$

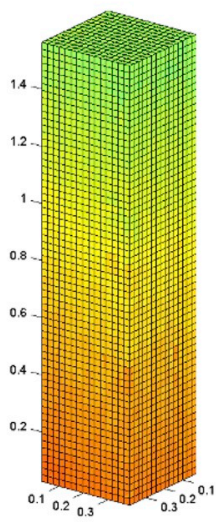

(g) $170 \mathrm{~N}$

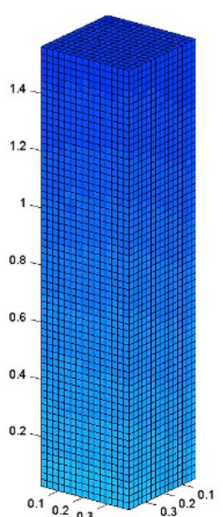

(c) $120 \mathrm{~N}$

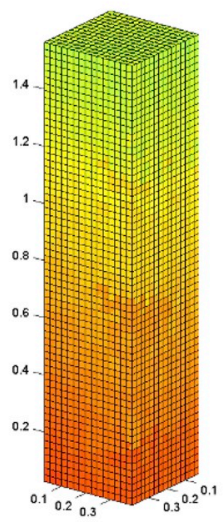

(h) $180 \mathrm{~N}$

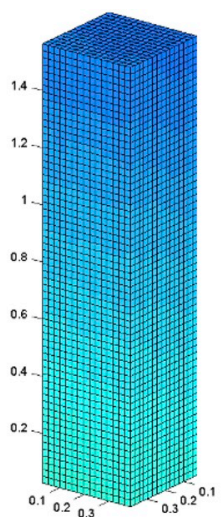

(d) $140 \mathrm{~N}$
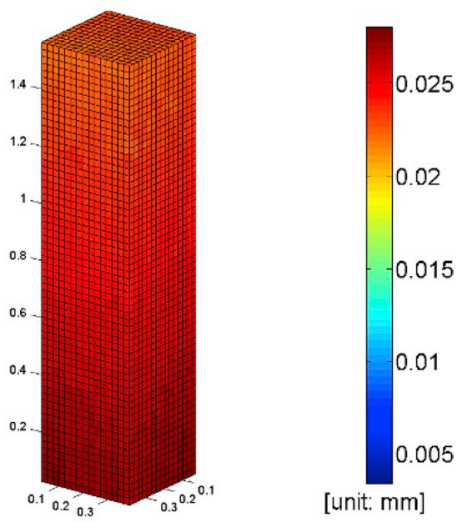

(i) $190 \mathrm{~N}$

Fig. 10. DVC analysis results at various loading steps. The loading steps are associated with the stress-strain curve shown in Fig. 11.

and

A $\quad a_{1}, a_{2, \ldots, a_{10}}{ }^{T}$

The unknown coefficients can be found from Eq. (4) by first multiplying the both sides with the transpose of $\mathbf{X}$ and then multiplying the both sides again with $\mathbf{X}^{\mathbf{T}} \mathbf{X}$ :

$$
\text { A } \quad \mathbf{X}^{\mathbf{T}} \mathbf{X} \quad{ }^{1} \mathbf{X}^{\mathbf{T}} \mathbf{u}
$$

Now the peak point on the surface expressed in Eq. (3) can be found at which the following derivatives of $C$ vanish;

$$
\begin{array}{lllll}
\frac{\partial C}{\partial x} & a_{2} & a_{5} y & a_{7} z & 2 a_{8} x \\
\frac{\partial C}{\partial y} & a_{3} & a_{5} x & a_{6} z & 2 a_{9} y \\
\frac{\partial C}{\partial z} & a_{4} & a_{6} y & a_{7} x & 2 a_{10} z
\end{array}
$$

Eq. (9) is another system of linear equations that can be solved for $x, y$, and $z$, i.e., the coordinates of the new correlation point.

\subsection{Computation of strain fields}

Strain fields can be obtained once the displacement fields are fully 


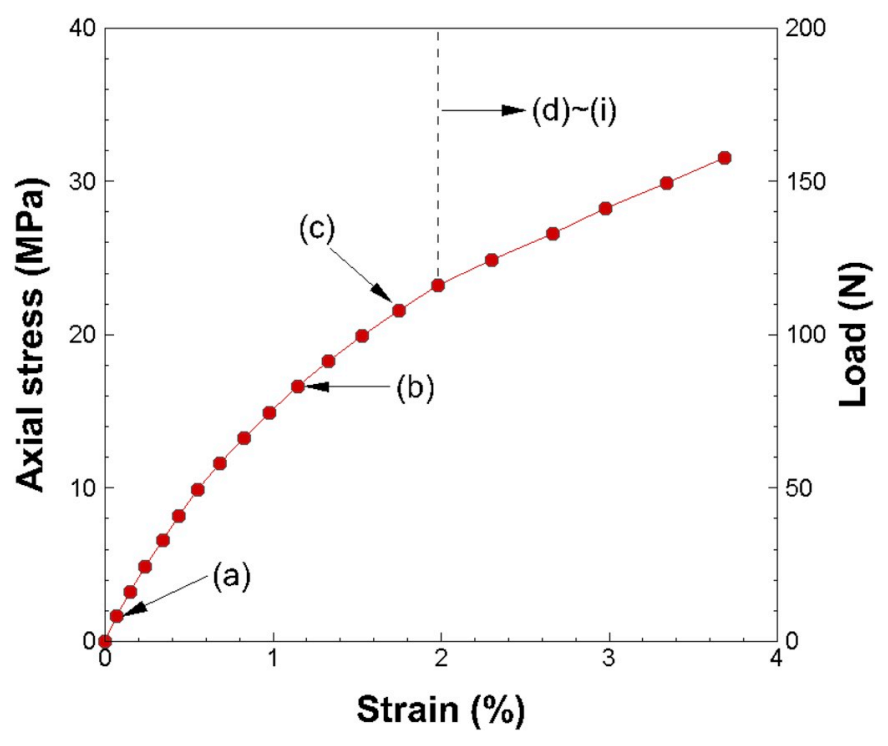

Fig. 11. Stress-strain curve of the polymer. The circles indicate the loading steps at which the X-ray images are taken. DVC analysis are performed at each loading step and the results are partly shown in Fig. 10.

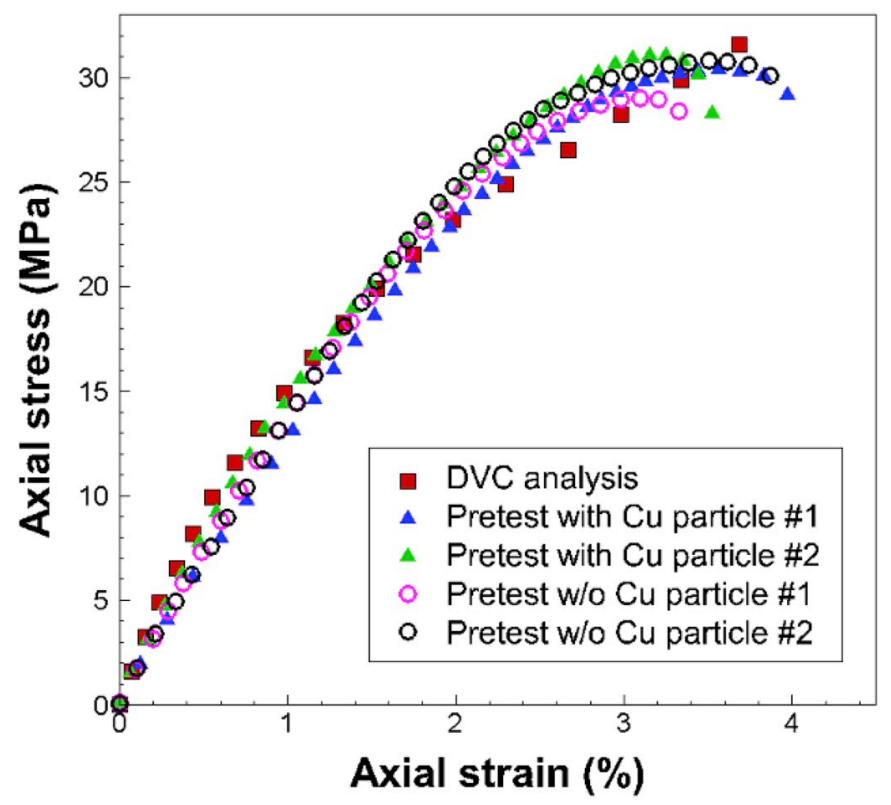

Fig. 12. Comparison of DVC analysis results against experimental data.

identified from the DVC analysis. Strain is a measure of how rapidly the displacement changes in a deforming material and thus defined with partial derivatives of displacement fields or a displacement gradient. In the present analysis, the deformation gradient is effectively computed using a well-established finite element (FE) approach. The main idea is to consider the center points of subvolumes as nodal points of a local element of an FE model. DOFs of every node are transferred from the results of the DVC analysis. Fig. 9 illustrates the process of constructing such a local element using the center points of subvolumes in the case of a 2D (two dimensional) FE model. As shown in Fig. 9, a four-node quadrilateral element is constructed with the center points of the four neighboring facets. Since the element already has the displacement data at each node, strains can easily be computed using the conventional FE approach based on the Lagrange shape functions. For 3D volume data, an eight-node hexahedron element can be constructed. Again, the DOFs at each node of the six-sided element are provided from the DVC

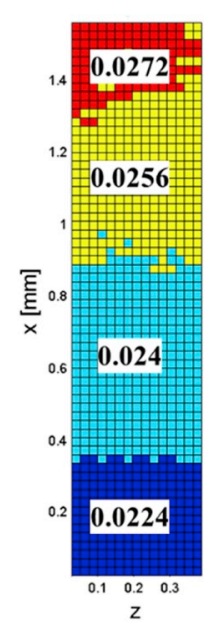

(a)

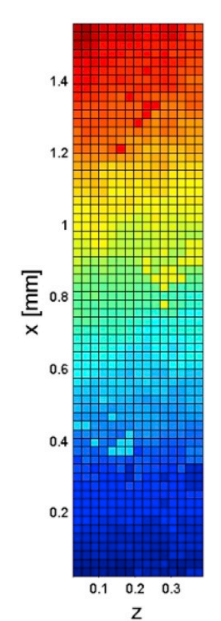

(b)
Fig. 13. Loading directional displacement field at $190 \mathrm{~N}$ (a) Displacement field obtained without the sub-voxel registration method (Note that numbers inside four textboxes denote the displacement [mm] of corresponding regions). (b) Displacement field obtained with the sub-voxel registration algorithm.

analysis.

\section{Results and discussions}

\subsection{Displacement fields from DVC analysis}

The present DVC analysis is demonstrated with the CT data obtained from the in situ tensile tests described in the prior section. Partial region of the specimen is selected for the deformation analysis to save computational time and cost as indicated in Fig. 5(b). The cubicle volume of interest (VOI), located at the center of the specimen, has the size

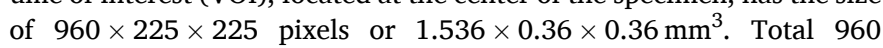
tomographic images are stacked in the VOI space along the loading direction. The VOI is discretized with 12,348 cubical subvolumes, where the size of one subvolume is $31 \quad 31 \quad 31$ pixels. The subvolumes are evenly distributed over the region, and each subvolume is overlapped with adjacent ones by 15 pixels in the $x, y$, and $z$ directions.

Fig. 10 shows the loading-directional displacement field computed through the present DVC analysis at several loading steps with a uniform interval of $10 \mathrm{~N}$. For all the loading steps, the average correlation coefficient of all the subvolumes inside the VOI is 0.7532 . This high correlation coefficient value indicates that the position tracking of all the subvolumes in the VOI are fairly successful. Position changes of all the subvolumes result in the displacement field of the sample, which is mapped on the undeformed configuration of the VOI as shown in Fig. 10. The displacement field is continuously increasing as the load increases from Fig. 10 (a) thru (b). Note that the top of the specimen is fixed and the bottom is pulled down in the experiment. Fig. 11 shows the stressstrain response of the epoxy material. Each circle on the curve indicates the loading steps at which X-ray CT scanning is carried out. The stresses in Fig. 11 are computed by dividing the loads measured from the load cell by the initial cross-sectional area of the sample while the strains are obtained by dividing the cross-head displacement with the initial gage length. The loading steps of the results displayed in Fig. 10 are also indicated in Fig. 11.

Fig. 12 compares the results of the present DVC analysis against the experimental data obtained from preliminary tests performed prior to the in situ tensile test. Specimens of the same configuration, but with and without the copper particles, are fabricated for the pretests. All these tension tests are conducted using the same loading device used for the in situ test for the sake of consistency. Loading is continuously applied to 


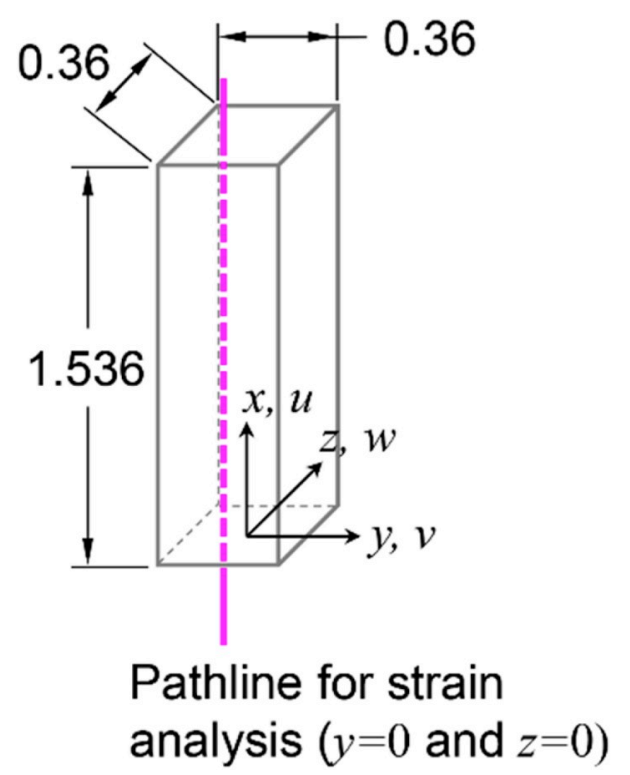

Fig. 14. Example of the definition of a pathline with the VOI dimensions (unit: millimeters).

the specimens without any interruption in the pretests. The vertical axis in Fig. 12 represents the loading directional engineering stresses obtained by dividing the measured load with the initial cross-sectional area of a specimen. The axial strains from the pretests are defined as the crosshead displacement divided by the gauge length of a specimen. As can be seen in Fig. 12, the particles have a negligible effect on the global response of the material. It is reiterated here that the particles are embedded into the epoxy sample to introduce the random distribution of grayscale values in the CT images, not to enhance the mechanical properties of the polymeric material. The DVC strains at each loading step in Fig. 12 are the average values of the strains in the VOI computed using the FEM approach described in the previous section. As shown in Fig. 12, the DVC results agree reasonably well with the experimental data. The DVC results slightly deviate from the test data in the plastic regime. The discrepancy is mainly due to the differences between the definitions of the strain. The experimental strains are defined in a global sense to include all the nonlinearities within the gage length while the DVC strains are computed in a very small local region.

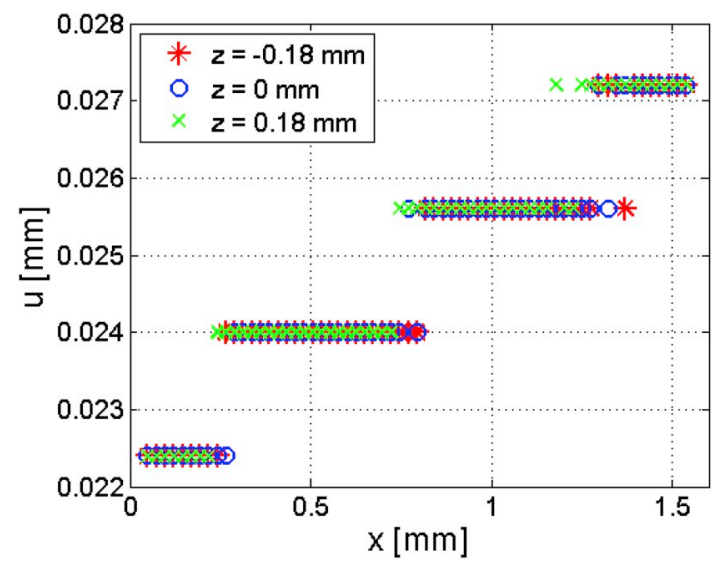

(a)

\subsection{Demonstration of the sub-voxel registration algorithm}

In this section, the effect and necessity of implementing the sub-voxel registration algorithm into the DVC analysis is demonstrated. For an example of the demonstration, the DVC results at $190 \mathrm{~N}$ in Fig. 12 are utilized here. Fig. 13 compares the loading-direction displacement with and without the sub-voxel registration approach. As shown in Fig. 13(a), the displacement values calculated at the maximum resolution of one pixel are unrealistically invariant in a number of wide areas. The differences in the displacement between two neighboring regions are $0.0016 \mathrm{~mm}$, which is the physical length of one pixel in the present study. Fig. 13(b) shows that the maximum resolution limit is overcome by the implementation of the sub-voxel registration method.

The importance of sub-voxel resolution in computing the displacement field is highlighted in Fig. 15 more specifically. Fig. 15 show the calculated displacement values along the pathlines defined in Fig. 14. Displacement data computed along the three straight lines defined at $z$

$18 \mathrm{~mm}, z \quad 0$ and $z \quad 18 \mathrm{~mm}$ with $y \quad 0$ are utilized to study the effect of the sub-voxel resolution and they are plotted in Fig. 15. As can be seen in Fig. 15(a), the DVC analysis without the capability of matching at the sub-voxel resolution exhibits piecewise constant results. When there exists no gradient in the displacement field, zero strains are computed in the wide region, which is an unacceptable measurement for the continuum material considered in the present study. The DVC analysis with the sub-voxel registration is capable of measuring reasonable deformation behavior of the sample as shown in Fig. 15(b). The displacement data computed along the three different pathlines are fairly consistent with each other. Furthermore, it is obvious that the discrete data points can easily be represented by a linear function. This implies nearly constant axial strains in the VOI region, which is expected from the present simple tensile test. The comparison results in Fig. 15 demonstrate the necessity of the sub-voxel registration technique especially for the computation of strains.

\section{Conclusions}

We have presented the protocol of the DVC analysis of a polymer material with synchrotron-based X-ray tomography. The epoxy material is first embedded with high-density particles to introduce a 3D speckle pattern inside the material. Copper particles are utilized in the present study. The particles create the random distribution of grayscales values in the CT images, which is necessary for the following image analysis. In situ tensile testing is performed at a synchrotron facility to obtain 3D volume images of the sample at multiple loading steps. The 3D images are used for the ZNCC-based DVC analysis to identify the deformation

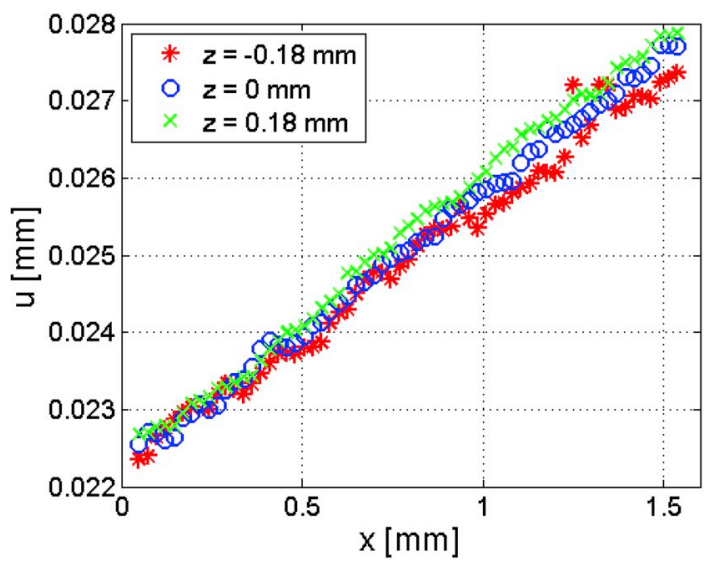

(b)

Fig. 15. (a) Displacement values computed from the DVC analysis without the sub-voxel registration method (b) DVC results along the pathlines with the sub-voxel registration approach. 
behavior inside the material. The fundamental principle of the DVC approach is to track the location changes of a number of cubic blocks between the reference and deformed images. In order to efficiently perform the recursive correlation operations, we have implemented various methodologies in addition to the classical FFT and precomputation approaches. The peak finding method refines the correlation resolution lower than a one-pixel size. The present DVC technique is demonstrated on the polymer material under tension and good agreements between the DVC results and experimental data are shown. The present study can be instrumental for future DVC analysis of a polymer material with a more complex configuration, e.g., quantitative analysis of the effect of voids on subsurface fracture behavior.

\section{Data availability}

The raw/processed data required to reproduce these findings cannot be shared at this time as the data also forms part of an ongoing study.

\section{Acknowledgement}

The authors are grateful for the financial support from UNIST (Ulsan National Institute of Science and Technology) through the 2019 Research Fund (1.90009.01). This work is also supported by Basic Science Research Program through the National Research Foundation of Korea (NRF) funded by the Ministry of Education (Grant No.: 2017R1D1A1B03035011). The authors extend their sincere gratitude to Dr. Jaehong Lim at PAL for his support in the in situ test.

\section{Appendix}

\section{A. Fast Fourier transform approach}

Fast Fourier transform (FFT) is commonly utilized for the DIC approach to improve its computational efficiency [34]. Before applying FFT to the DVC process, the numerator of Eq. (2) is rewritten as

$$
\begin{aligned}
& \sum_{i}^{N_{x}} \sum_{j}^{N_{y}} \sum_{k=1}^{N_{z}} f x_{i}, y_{j}, z_{k} \quad f_{0} \quad g \quad x_{i} \quad u_{I}, y_{j} \quad v_{J}, z_{k} \quad w_{K} \quad g_{0} \\
& \sum \sum \sum f \times g^{\prime} \mathbf{x} \quad \mathbf{p} \quad f_{0} \sum \sum \sum g^{\prime} \mathbf{x} \quad \mathbf{p}
\end{aligned}
$$

where $g^{\prime} \mathbf{x} \quad \mathbf{p} \quad g \mathbf{x} \quad \mathbf{p} \quad g_{0}, \mathbf{x} \quad x_{i}, y_{j}, z_{k}$ and $\mathbf{p}$ is the displacement vector. In the last term of Eq. (A1),

$$
\begin{array}{ccccc}
\sum \sum \sum g^{\prime} \mathbf{x} & \mathbf{p} & \sum \sum \sum g \mathbf{x} & \mathbf{p} & g_{0} \\
\sum \sum \sum g \mathbf{x} & \mathbf{p} & N_{x} N_{y} N_{z} g_{0} & & \\
\frac{\sum \sum \sum g \mathbf{x}}{N_{x} N_{y} N_{z}} & & g_{0} \quad N_{x} N_{y} N_{z} & 0
\end{array}
$$

The right hand side of Eq. (A1) is thus written as

$$
\sum \sum \sum f \mathbf{x} g \mathbf{x} \quad \mathbf{p} \quad g_{0} \sum \sum \sum f \mathbf{x}
$$

The first term of Eq. (A3) can be considered as the typical expression of the discrete cross-correlation with the real-valued function $f$. Indeed, when the first term of Eq. (A3) is normalized by the standard deviations in a similar manner as Eqs. (1) and (2), it defines the correlation coefficient of the normalized cross-correlation (NCC) method. The cross-correlation between the two functions $f \mathbf{x}$ and $g \mathbf{x}$ is simply defined as the convolution of $\bar{f} \quad \mathbf{x}$ and $g \mathbf{x}$ where $\bar{f}$ denotes the complex conjugate of $f$ [39]:

$\sum \sum \sum f \mathbf{x} g \mathbf{x} \quad \mathbf{p} \quad f \star g \quad \bar{f} \quad \mathbf{x} * g \mathbf{x}$

According to the convolution theorem, Eq. (A4) can be expressed using the Fourier transform.

$\bar{f} \quad \mathbf{x} * g \mathbf{x} \quad F^{1}\left\{F\left\{\boldsymbol{f}^{\prime}\right\} \cdot F\{\boldsymbol{g}\}\right\}$

where $F$ denotes the Fourier transform operator. $f$ is a $N_{x} \quad N_{y} \quad N_{z}$ matrix for which $f$ matrix is reflected with respect to the $x, y$, and $z$ directions when $f$ is the grayscale matrix of the reference subvolume. $g$ is a $N_{x} \quad N_{y} \quad N_{z}$ matrix representing the intensity of the comparing subvolume. The dot operator in Eq. (A5) denotes point-wise multiplication.

Suppose that the size of the searching region is $M_{x} \quad M_{y} \quad M_{z}$. If the 3D cross-correlation is performed in spatial domain using Eq. (A3), the

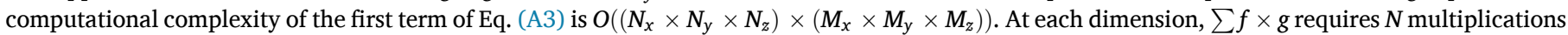

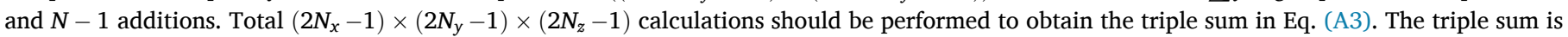

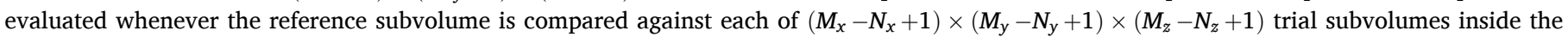
searching region. When the correlation process is performed in frequency domain with an FFT algorithm, Eq. (A5) should be first extended to include the entire searching region such that

$F^{1}\left\{F\left\{\boldsymbol{F}^{\prime}\right\} \cdot F\{\boldsymbol{G}\}\right\}$

where $\boldsymbol{G}$ is the intensity matrix of the searching region (see Fig. 6) and $\boldsymbol{F}^{\prime}$ is a zero-padded matrix of $\boldsymbol{f}^{\prime}$ to have the same size with the $\boldsymbol{G}$ matrix. Then, the required computational cost based on Eq. (A6) is $O \quad M_{x} \quad M_{y} \quad M_{z} \quad \log M_{x} \quad M_{y} \quad M_{z} \quad$ for the Fourier transform, $O M_{x} \quad M_{y} \quad M_{z}$ for the point- 
wise multiplications and $O \quad M_{x} \quad M_{y} \quad M_{z} \quad \log M_{x} \quad M_{y} \quad M_{z} \quad$ for the inverse Fourier transform. The FFT approach to compute the first term of Eq. (A3) becomes relatively more efficient as the subvolume size approaches the searching region and both of them are sufficiently large [34].

B. Precomputation for summing operations

In order to utilize FFT as described in Appendix A, Eq. (2) has been transformed into

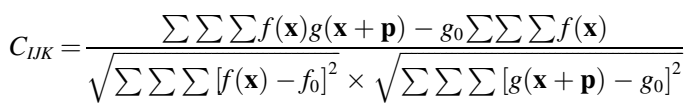

In addition to the FFT approach, precomputation strategy is employed to further improve the computing efficiency of the correlation coefficient, following the approach suggested by Lewis [34]. The main idea is to pre-calculate summations in Eq. (B1) and allocate memory to store the data for future usage $[18,34,35]$. It is obvious that summations associated with the reference subvolume in Eq. (B1) need to be evaluated only once during the recursive operation to determine all $C_{I J K}$ over an entire searching region. They are $\sum \sum \sum f \mathbf{x}$ and $\sum \sum \sum f \mathbf{x} \quad f_{0}{ }^{2}$ in the numerator and denominator of Eq. (B1), respectively. Furthermore, $\sum \sum \sum f \mathbf{x} \quad f_{0}{ }^{2}$ can be efficiently computed if the summation of $\sum \sum \sum f$ is conducted in advance and the data is utilized with $\sum \sum \sum f^{2}$. Lastly, the term associated with $g$ in the denominator of Eq. (B1) can also be efficiently computed. The square term is expanded as

$\sum \sum \sum g \mathbf{x} \quad \mathbf{p} \quad g_{0}{ }^{2} \quad \sum \sum \sum g \mathbf{x} \quad \mathbf{p}^{2} \quad 2 g_{0} \sum \sum \sum g \mathbf{x} \quad \mathbf{p} \quad N_{x} N_{y} N_{z} g_{0}^{2}$

The intensity average $g_{0}$ can be expressed in terms of $g \mathbf{x} \quad \mathbf{p}$ such that

$g_{0} \frac{1}{N_{x} N_{y} N_{z}} \sum \sum \sum g \mathbf{x} \quad \mathbf{p}$

The right-hand side of Eq. (B2) is then rewritten as

$\sum \sum \sum g \mathbf{x} \quad \mathbf{p}^{2} \frac{1}{N_{x} N_{y} N_{z}}\left\{\sum \sum \sum g \mathbf{x} \quad \mathbf{p}\right\}^{2}$

Again, similarly with the summations associated with $f$, precomputed data of $\sum \sum \sum g$ and $\sum \sum \sum g^{2}$ can save computational time and cost significantly [18]. In summary, the first term in the denominator of Eq. (B1) is computed using the FFT approach and the rest of summations is obtained from the precomputed data.

\section{References}

[1] M.A. Sutton, W.J. Wolters, W.H. Peters, W.F. Ranson, S.R. McNeill, Determination of displacements using an improved digital correlation method, Image Vis Comput. 1 (3) (1983) 133-139.

[2] H.A. Bruck, S.R. McNeill, M.A. Sutton, W.H. Peters, Digital image correlation using Newton-Raphson method of partial differential correction, Exp. Mech. 29 (3) (1989) 261-267.

[3] G. Vendroux, W.G. Knauss, Submicron deformation field measurements: Part 2. Improved digital image correlation, Exp. Mech. 38 (2) (1998) 86-92.

[4] F. Laraba-Abbes, P. Ienny, R. Piques, A new 'tailor-made' methodology for the mechanical behaviour analysis of rubber-like materials: I. Kinematics measurements using a digital speckle extensometry, Polymer 44 (3) (2003) $807-820$.

[5] M. Jerabek, Z. Major, R.W. Lang, Strain determination of polymeric materials using digital image correlation, Polym. Test. 29 (3) (2010) 407-416.

[6] H.W. Schreier, D. Garcia, M.A. Sutton, Advances in light microscope stereo vision, Exp. Mech. 44 (3) (2004) 278-288.

[7] M.A. Sutton, J.H. Yan, X. Deng, C.S. Cheng, P. Zavattieri, Three-dimensional digital image correlation to quantify deformation and crack-opening displacement in ductile aluminum under mixed-mode I/III loading, Opt. Eng. 46 (5) (2007), 051003.

[8] R. Guastavino, P. Goransson, A 3D displacement measurement methodology for anisotropic porous celluar foam materials, Polym. Test. 26 (6) (2007) 711-719.

[9] J. Engqvist, M. Wallin, S.A. Hall, M. Ristinmaa, T.S. Plivelic, Measurement of multiscale deformation of polycarbonate using X-ray scattering with in-situ loading and digital image correlation, Polymer 82 (15) (2016) 190-197.

[10] N. Candau, C. Pradille, J.L. Bouvard, N. Billon, On the use of a four-camera stereovision system to characterize large 3D deformation in elastomers, Polym. Test. 56 (2016) 314-320.

[11] A. Germaneau, P. Doumalin, J.C. Dupre, Comparison between X-ray microcomputed tomography and optical scanning tomography for full 3D strain measurement by digital volume correlation, NDT E Int. 41 (6) (2008) 407-415.

[12] B.K. Bay, T.S. Smith, D.P. Fyhrie, M. Saad, Digital volume correlation: threedimensional strain mapping using X-ray tomography, Exp. Mech. 39 (3) (1999) $217-226$.

[13] T.S. Smith, B.K. Bay, M.M. Rashid, Digital volume correlation including rotational degrees of freedom during minimization, Exp. Mech. 42 (3) (2002) 272-278.

[14] H. Schreier, J.J. Orteu, M.A. Sutton, Image Correlation for Shape, Motion and Deformation Measurements, Springer, New York, NY, United States, 2009.
[15] T. Wang, Z. Jiang, Q. Kemao, F. Lin, S.H. Soon, GPU accelerated digital volume correlation, Exp. Mech. 56 (2) (2016) 297-309.

[16] R. Zauel, Y.N. Yeni, B.K. Bay, X.N. Dong, D.P. Fyhrie, Comparison of the linear finite element prediction of deformation and strain of human cancellous bone to 3D digital volume correlation measurements, J. Biomech. Eng. 128 (1) (2006) 1-6.

[17] C. Franck, S. Hong, S.A. Maskarinec, D.A. Tirrell, G. Ravichandran, Threedimensional full-field measurements of large deformations in soft materials using confocal microscopy and digital volume correlation, Exp. Mech. 47 (3) (2007) $427-438$.

[18] J. Huang, X. Pan, S. Li, X. Peng, C. Xiong, J. Fang, A digital volume correlation technique for 3-D deformation measurements of soft gels, International Journal of Applied Mechanics 3 (02) (2011) 335-354.

[19] F. Forsberg, M. Sjodahl, R. Mooser, E. Hack, P. Wyss, Full Three-dimensional strain measurements on wood exposed to three-point bending: analysis by use of digital volume correlation applied to synchrotron radiation micro-computed tomography image data, Strain 46 (1) (2010) 47-60.

[20] S.A. Hall, M. Bornert, J. Desrues, Y. Pannier, N. Lenoir, G. Viggiani, P. Besuelle, Discrete and continuum analysis of localised deformation in sand using X-ray CT and volumetric digital image correlation, Geotechnique 60 (5) (2010) 315-320.

[21] J. Marrow, C. Reinhard, Y. Vertyagina, L. Saucedo-Mora, D. Collins, M. Mostafavi, 3D studies of damage by combined X-ray tomography and digital volume correlation, Procedia Materials Science 3 (2014) 1554-1559.

[22] INF-114/INF-212 Technical Data Sheet, Pro-Set Inc., Bay City, MI, USA, Retrieved February 21, 2019 from https://www.prosetepoxy.com/standard-products/ infusion-epoxies/.

[23] R. Brault, A. Germaneau, J.C. Dupre, P. Doumalin, S. Mistou, M. Fazzini, In-situ analysis of laminated composite materials by X-ray micro-computed tomography and digital volume correlation, Exp. Mech. 53 (7) (2013) 1143-1151.

[24] A.W. Nienow, M.F. EDWARDS, N. Harnby, Mixing in the Process Industries, second ed., Butterworth-Heinemann, Oxford, United Kingdom, 1997.

[25] J.H. Lim, S.J. Seo, H.Y. Kim, C.K. Ryu, S. Rah, J.Y. Huang, C.S. Lee, I.D. Seo, S. G. Kim, D. Lee, M.H. Cho, "Station for structural studies on macro objects: beamline 6C Bio medical imaging at the Pohang light source-II, Biodesign 5 (2) (2017) 53-61.

[26] M.A. Sutton, S.R. McNeill, J.D. Helm, Y.J. Chao, Advances in two-dimensional and three-dimensional computer vision, Photomechanics 77 (2000) 323-372.

[27] B. Pan, K. Qian, H. Xie, A. Asundi, Two-dimensional digital image correlation for in-plane displacement and strain measurement: a review, Meas. Sci. Technol. 20 (6) (2009) 062001.

[28] A. Giachetti, Matching techniques to compute image motion, Image Vis Comput. 18 (3) (2000) 247-260. 
[29] W. Tong, An evaluation of digital image correlation criteria for strain mapping applications, Strain 41 (4) (2005) 167-175.

[30] B. Pan, H. Xie, Z. Wang, Equivalence of digital image correlation criteria for pattern matching, Appl. Opt. 49 (28) (2010) 5501-5509.

[31] B. Pan, Z. Wang, H. Xie, Generalized spatial-gradient-based digital image correlation for displacement and shape measurement with subpixel accuracy, J. Strain Anal. Eng. Des. 44 (8) (2009) 659-669.

[32] B. Pan, K. Li, A fast digital image correlation method for deformation measurement, Opt. Lasers Eng. 49 (7) (2011) 841-847.

[33] E. Bar-Kochba, J. Toyjanova, E. Andrews, K.S. Kim, C. Franck, A fast iterative digital volume correlation algorithm for large deformations, Exp. Mech. 55 (1) (2015) 261-274.

[34] J.P. Lewis, Fast template matching, in: D. Laurendeau, C. Cedras (Eds.), Proceedings of Vision Interface 95, Quebec City, Canada, May 15-19, Canadian Image Processing and Pattern Recognition Society, Canada, 1995, pp. 120-123.

[35] K. Briechle, U.D. Hanebeck, Template matching using fast normalized cross correlation, in: D.P. Casasent, T. Chao (Eds.), Proceedings of SPIE Vol. 4387; Optical Pattern Recognition XII, Orlando, FL, United States, March 16-20, 2001, Society of Photo Optical, 2001.

[36] E. Verhulp, B. van Rietbergen, R. Huiskes, A three-dimensional digital image correlation technique for strain measurements in microstructures, J. Biomech. 37 (9) (2004) 1313-1320.
[37] M.R. Hardisty, C.M. Whyne, Whole bone strain quantification by image registration: a validation study, J. Biomech. Eng. 131 (6) (2009) 064502.

[38] B.C. Roberts, E. Perilli, K.J. Reynolds, Application of the digital volume correlation technique for the measurement of displacement and strain fields in bone: a literature review, J. Biomech. 47 (5) (2014) 923-934.

[39] R. Bracewell, Fourier Analysis and Imaging, Springer-Science Business Media, LLC., New York, NY, United States, 2003.

[40] P. Bing, X. Hui-Min, X. Bo-Qin, D. Fu-Long, Performance of sub-pixel registration algorithms in digital image correlation, Meas. Sci. Technol. 17 (6) (2006) 1615.

[41] P. Zhou, K.E. Goodson, Subpixel displacement and deformation gradient measurement using digital image/speckle correlation, Opt. Eng. 40 (8) (2001) 1613-1621.

[42] C.Q. Davis, D.M. Freeman, Statistics of subpixel registration algorithms based on spatiotemporal gradients or block matching, Opt. Eng. 37 (4) (1998) 1290-1299.

[43] B.D. Lucas, T. Kanade, An iterative image registration technique with an application to stereo vision, in: A. Drinan (Ed.), Proceedings of the 7th International Joint Conference on Artificial Intelligence, Vancouver, British Columbia, Canada, August 24-28, 1981, Morgan Kaufmann Publishers Inc., San Francisco, CA, United States, 1981, pp. 674-679. 